\title{
PREVALENCIA Y CAPACIDAD DE RECONOCIMIENTO DEL PLAGIO ACADEMICO ENTRE EL ALUMNADO DEL ÁREA DE ECONOMÍA
}

\author{
Prevalence and ability to recognize academic \\ plagiarism among university students in economics
}

Rubén Comas-Forgas y Jaume Sureda-Negre
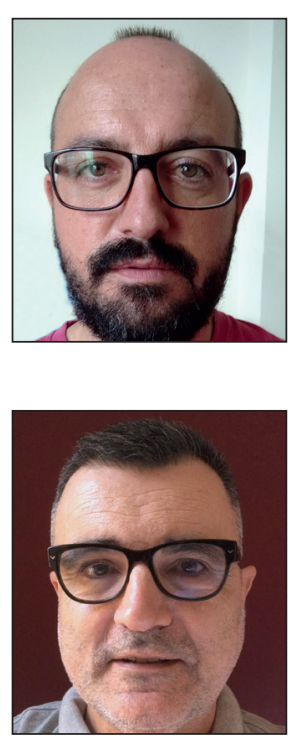

Rubén Comas-Forgas es doctor europeo en ciencias de la educación por la Universidad de las Islas Baleares donde actualmente es profesor adscrito al Departamento de Pedagogía Aplicada y Psicología de la Educación. Sus líneas de trabajo se centran en la integridad académica y la documentación científica en ciencias de la educación. Ha sido investigador/profesor visitante en diversos centros como: University of East Anglia (Reino Unido), Liverpool John Moores University (Reino Unido), Panteion University of Social and Political Sciences (Grecia), y Universidad Autónoma de Yucatán (México).

http://orcid.org/0000-0002-8885-753X

rubencomas@uib.es

Jaume Sureda-Negre es catedrático de universidad y director del Departamento de Pedagogía Aplicada y Psicología de la Educación de la Universidad de las Islas Baleares. Ha ocupado durante varios años cargos de gestión académica (vicerrectorados y dirección de institutos de investigación). Ha dirigido numerosos proyectos de investigación competitivos tanto a nivel nacional como internacional. Sus líneas de trabajo se centran en la integridad académica y la documentación científica en ciencias de la educación.

http://orcid.org/0000-0003-2689-5626

sureda.negre@gmail.com

Universidad de las Islas Baleares Campus UIB, Edificio Beatriu Pinos, 16. 01722 Palma de Mallorca, España

\section{Resumen}

Se estudia la relación existente entre el conocimiento de lo que supone plagio académico y el conocimiento de las normas de citación, y la comisión de plagio entre alumnado de último curso del área de Economía en una universidad de tamaño medio española. Para ello se contó con la participación de una muestra $(n=325)$ de alumnos y alumnas a quienes se les administró un cuestionario elaborado ad-hoc para este estudio. Esta investigación pone de manifiesto los déficits que tiene el alumnado participante para determinar cuándo un texto está plagiado y qué es una cita y una referencia. Los resultados sugieren la inexistencia de relación entre el reconocimiento de lo que supone un plagio y la prevalencia en su comisión. El estudio aporta datos relevantes acerca del alumnado que comete plagio de forma accidental y aquél que lo hace intencionalmente.

\section{Palabras clave}

Competencias informacionales; Plagio académico; Integridad académica; Evaluación de competencias; Normas de citación.

\section{Abstract}

The relationship between knowledge of what constitutes academic plagiarism, knowledge of citation norms, and academic plagiarism perpetration among students in their final year of Economics at an average size Spanish university is analyzed. The research counted on the participation of a sample $(n=325)$ of students who responded to a questionnaire prepared adhoc for this study. This research highlights the deficits that participating students had in determining when a text is plagiarized and in recognizing a citation or a reference. The results suggest an absence in students' ability to recognize plagiarism relates directly to the commission of it. The study provides data on students who commit plagiarism accidentally and those who do it intentionally.

\section{Keywords}

Information competencies; Academic plagiarism; Academic integrity; Competencies assessment; Citation norms. 
Comas-Forgas, Rubén; Sureda-Negre, Jaume (2016). “Prevalencia y capacidad de reconocimiento del plagio académico entre el alumnado del área de economía". El profesional de la información, v. 25, n. 4, pp. 616-622.

http://dx.doi.org/10.3145/epi.2016.jul.11

\section{Introducción}

La comunicación académica debe regirse, entre otros, por los principios de honestidad, veracidad, rigor, calidad, probidad e integridad. La contravención de dichos valores supone la comisión de prácticas deshonestas y fraudulentas. Engañar, falsificar, defraudar, mentir, adulterar, ocultar, confundir, etc., son verbos no conjugables en la transmisión e intercambio de conocimiento en el campo educativo.

A pesar de ello son numerosas las señales e indicios que advierten que las prácticas deshonestas están ciertamente asentadas en las universidades (Hallak; Poisson, 2002; Poisson, 2010). Y no sólo eso, el problema es todavía más alarmante ya que parece existir cierto acuerdo en cuanto a que los usos y praxis fraudulentas van en aumento en lugar de retroceder (Hallak; Poisson, 2007; Christakis; Christakis, 2012).

La modalidad de fraude en las actividades de evaluación más extendida entre el alumnado es, sin duda alguna, la comisión de plagio académico. Por ejemplo, centrándonos en España, según los escasos estudios llevados a cabo sobre la materia, más de la mitad de los universitarios españoles (cifras en torno al 60\%) manifiesta haber incidido en alguna modalidad de plagio académico a la hora de redactar trabajos para asignaturas de sus estudios (Comas-Forgas, 2009; Comas-Forgas; Sureda-Negre; Salva-Mut, 2010).

$Y$ este hecho, en bastantes ocasiones, se ha relacionado con un déficit de competencias informacionales entre el alumnado -por ejemplo: desconocimiento de normativas de citación y elaboración de referencias, incapacidad para reconocer lo que supone plagio de lo que no (Moon, 2002; Guedes; Gomes-Filho, 2015, DeGeeter et al., 2014)- lo que genera situaciones en las que el alumnado incurre en plagio de forma inconsciente o accidental (Park, 2003). A la vez, se ha demostrado que una adecuada formación en competencias informacionales actúa como factor de prevención y reducción de los casos de plagio académico entre alumnado (Hurlbert; Savidge; Laudenslager, 2003; Humphreys, 2013).

Ante esta situación conviene poner de manifiesto que unas pocas universidades españolas, desde sus servicios de biblioteca y documentación, ofrecen recursos formativos/ informativos al alumnado acerca de cómo eludir la comisión de plagio en la presentación de trabajos académicos (Domínguez-Aroca, 2012). Además, algunos centros universitarios españoles han introducido en sus planes de estudio asignaturas centradas en mejorar las competencias informacionales del alumno (búsqueda y localización de información, valoración de la información, citar y referenciar las fuentes, etc.). Sea como fuere, todavía no disponemos de datos y evidencias para a conocer el impacto de estas iniciativas en la mejora de las competencias del alumnado para producir trabajos académicos de calidad.
Las cuestiones de investigación $(\mathrm{Cl})$ que se intentan responder con esta propuesta son:

CI1: ¿Son capaces los alumnos de último curso del área de economía de reconocer un texto plagiado y diferenciarlo de un texto elaborado con arreglo a los principios y estándares académicos?

$\mathrm{Cl}$ 2: ¿Son capaces los alumnos de último curso del área de economía de reconocer qué es una cita y una referencia bibliográfica?

CI3: ¿Cuál es el nivel de prevalencia en la comisión de plagio académico entre el alumnado de último curso del área de economía?

C31: ¿Existen diferencias significativas entre el alumnado de último curso del área de economía en cuanto a la frecuencia de comisión de plagio académico atendiendo al género?

C32: ¿Existen diferencias significativas entre el alumnado de último curso del área de economía en cuanto a la frecuencia de comisión de plagio académico atendiendo a su capacidad para reconocerlo?

C33: ¿Existen diferencias significativas en cuanto a la frecuencia de comisión de plagio académico atendiendo al conocimiento que posee el alumno de lo que son las citas y las referencias bibliográficas?

\section{Material y método}

\subsection{Población y muestra}

En el estudio han participado 325 estudiantes de cuarto curso, de los años 2013-2014 y 2014-2015, del grado en administración y dirección de empresas (GADE) y del grado de economía (GECO) de la Universidad de las Islas Baleares. Ello representa una muestra con un margen de error, calculado para el total de alumnado matriculado en cuarto curso de las dos titulaciones en ambos años académicos ( $n=492)$, de $\pm 3,17 \%$ para un intervalo de confianza estimado del $95 \%$ bajo la condición más desfavorable de $p=q=0,50$. El alumnado de estas titulaciones no ha contado en su formación con ninguna asignatura estrictamente dedicada a mejorar sus competencias informacionales.

La recogida de datos se realizó, de manera individual y anónima en situaciones de aula con la presencia del equipo de investigadores, en dos tandas: enero de 2014 (alumnado del curso 2013-14) y enero de 2015 (alumnado del curso 201415). Ningún alumno rehusó participar en el estudio; sin embargo, pese a que se obtuvo un total de 329 encuestas, la muestra se contrajo hasta 325 sujetos, dado que 4 cuestionarios fueron invalidados por alguno de estos motivos:

- multiplicidad de respuesta en items de respuesta única;

- cuestionarios contestados en un porcentaje inferior al 
$50 \%$ de los items;

- ininteligibilidad de las respuestas dadas por los encuestados.

En relación a las características de los sujetos:

- el 48,9\% fueron mujeres y el 51,1\% hombres;

- accedieron a la universidad por distintas vías: 92,6\% a través de la Prueba de acceso a la universidad (PAU o Selectividad), 1,9\% a través de pruebas de acceso a mayores de 25 años, $4 \%$ a través de una titulación de FP de grado superior, y un $1,5 \%$ a través de otras titulaciones;

- en cuanto a la distribución por edades, éstas varían entre los 21 y los 48 , situándose la media en 23,7 años (con una desviación típica de 3,3).

\subsection{Fuente de datos y variables}

En la recolección de datos se empleó como instrumento el Cuestionario sobre plagio académico entre el alumnado de GADE y GECO, diseñado expresamente para el presente estudio y fundamentado en:

- análisis de la bibliografía existente sobre la materia;

- adaptación de diversos items de los cuestionarios validados de Roig (1997), Comas-Forgas (2009) y Morey (2011).

Este tipo de encuestas son los instrumentos más utilizados para el estudio de la integridad académica, presentando un aceptable nivel de fiabilidad (Cizek, 1999; Comas-Forgas, 2009). Constaba de 10 preguntas:

- cinco referidas a características del alumnado (género, edad, vía de acceso a los estudios, nota de acceso a la carrera y media de expediente académico);

- una sobre la formación recibida en elaboración y presentación de trabajos académicos;

- dos centradas en analizar el nivel de conocimientos respecto a las citas académicas y las referencias bibliográficas;

- una para determinar el nivel de conocimiento que tienen de lo que supone un texto plagiado; y

- una referida a la comisión de diversas prácticas constitutivas de plagio por parte del alumnado.

Los resultados que se publican en el presente trabajo se centran en el análisis de tres de las variables abordadas en el cuestionario y la posterior asociación entre éstas (V3 con V1, V2 y V4):

V1: Capacidad del alumnado para determinar cuándo un texto está plagiado.

V2: Conocimiento de la normativa de citación y elaboración de referencias bibliográficas.

V3: Frecuencia e índice de perpetración de plagio académico.

V4: Género.

Como indicador de medición de la V1, se adaptó y simplificó el cuestionario Plagiarism knowledge survey (PKS) de Roig (1997). Al igual que Roig, se presentaron a los participantes en el estudio diversos textos (4 en total). El primero era un fragmento extraído literalmente de la Wikipedia sobre la revolución francesa; se hacía saber a los participantes que se trataba un texto original y se les indicaba cuál era su fuente. El texto original se había transformado, por los investigadores, en otros tres textos:
1) Texto A: representaba un parafraseado del texto original con ausencia de cita;

2) Texto B: era un plagio literal del texto original ya que sólo se cambió el orden de las frases y no se citó la fuente;

3) Texto C: se trataba de un uso correcto de la información original, ya que incluían citas directas y parafraseados con citación de la fuente primaria.

Los participantes debían contestar, una vez leídos los cuatro textos, las siguientes preguntas:

- cuál de los tres fragmentos derivados del original supone un plagio;

- cuál de los tres fragmentos derivados del original te parece más adecuado para entregar en un trabajo académico;

- cuál de los tres fragmentos te parece menos adecuado para entregar en un trabajo académico.

Las posibilidades de respuesta para cada pregunta eran: "Texto A", Texto B", Texto C" y "No lo sé". De cara a poder establecer un índice de reconocimiento de plagio (en adelante, IRP), se codificaron las respuestas (3 puntos por cada respuesta correcta y 1 punto para las respuestas incorrectas) y se realizó el sumatorio para cada participante de la puntuación obtenida en cada pregunta:

- los alumnos con tres aciertos obtenían una puntuación de 9;

- los que contestaron acertadamente dos de las tres preguntas tenían una puntuación de 7;

- los que contestaron bien una de las tres preguntas sumaban 5 puntos;

- los que no contestaron correctamente ninguna de las preguntas de la V1 obtuvieron 3 puntos.

Para medir la V2 los investigadores emplearon dos preguntas adaptadas de Morey (2011):

a) una pregunta en la que se presentaba una definición de lo que es una cita y los participantes tenían 5 opciones de respuesta para contestar a qué hacía referencia la definición presentada ("Cita", "Nota a pie de página", "Referencia bibliográfica”, "Bibliografía” y "No lo sé");

b) una pregunta en la que se presentaba una referencia bibliográfica de un libro de un autor, elaborada según la normativa APA y los participantes debían contestar qué era, disponiendo de cinco opciones: "Nota a pie de página", "Paráfrasis bibliográfica", "Cita", "Referencia bibliográfica" y "No lo sé".

Con el fin de generar un índice de conocimiento de las citas y referencias bibliográficas (en adelante ICCRB) se codificaron las respuestas dadas ( 3 puntos por cada respuesta correcta y 1 punto las respuestas incorrectas) y se realizó el sumatorio para cada participante de la puntuación de cada pregunta:

- los alumnos con dos aciertos obtenían una puntuación de 6; - los que contestaron acertadamente una de las dos preguntas tenían una puntuación de 4;

- los que no contestaron correctamente ninguna de las preguntas obtuvieron 2 puntos.

La V3 se fundamenta en una escala creada por ComasForgas (2009) y mide las respuestas emitidas acerca de la 
frecuencia en la realización de 7 acciones constitutivas de plagio (planteadas de manera independiente):

- Acción 1: entregar un trabajo completo, realizado por el propio alumno, que ya había sido entregado en cursos o convocatorias anteriores.

- Acción 2: entregar un trabajo realizado por otro alumno que ya había sido entregado en cursos o convocatorias anteriores (para la misma asignatura o para otra).

- Acción 3: copiar de páginas web fragmentos de textos ysin citar- pegarlos directamente en un documento -en el cual hay una parte de texto escrita por el propio alumnoy entregarlo como trabajo de una asignatura.

- Acción 4: bajar o descargar un trabajo completo de internet y entregarlo, sin modificarlo, como trabajo propio de una asignatura.

- Acción 5: copiar fragmentos de fuentes impresas (libros, enciclopedias, periódicos, artículos de revista, etc.) y añadirlos -sin citar-como partes de un trabajo propio de una asignatura.

- Acción 6: hacer íntegramente un trabajo a partir de fragmentos copiados literalmente de webs (sin que ninguna parte del trabajo haya sido realmente escrita por el alumno/a).

- Acción 7: copiar partes de trabajos entregados durante cursos anteriores y, sin citar, usarlos como apartados de un trabajo nuevo.

Para cada acción, los participantes debían señalar la frecuencia con que habían efectuado este tipo de prácticas a lo largo del curso académico anterior al momento de realización del cuestionario. Podían escoger una de estas cinco posibilidades: "Nunca", "Entre 1 y 2 veces", "Entre 3 y 5 veces", "Entre 6 y 10 veces" o "Más de 10 veces". Las preguntas de la V3 se han recodificado para conseguir el índice de comisión de plagio académico (en adelante, ICPA) a partir del sumatorio de respuestas de cada alumno estableciéndose una escala comprendida entre 7 y 35 (donde 7 significa no haber plagiado y 35 representa haber plagiado muy frecuentemente).

\subsection{Análisis de los datos}

Para cada variable de categoría analizada se ha calculado la frecuencia y el porcentaje, mientras que en las variables de escala se ha calculado también la media, varianza y desviación típica. Seguidamente, con el objetivo de establecer potenciales asociaciones entre el ICPA y los índices IRP e ICCRB, se ha proyectado el análisis estadístico a partir de la comparación de medias, obtenida mediante la aplicación del análisis de la varianza anova. El análisis de la significación estadística en la asociación entre el género y los índices ICPA, IRP e ICCRB se ha efectuado mediante la prueba t-student para muestras independientes.

Para el conjunto de análisis llevados a cabo en el presente estudio se ha utilizado el paquete estadístico Statistical Package for the Social Sciences (SPSS, v. 19.0).

\section{Resultados}

\subsection{Reconocimiento de plagio}

Se recuerda al lector que los participantes debían leer tres fragmentos derivados de un texto original, extraído literalmente de Wikipedia, y determinar cuál de ellos era un plagio, cuál era la mejor opción para entregar como trabajo académico y cuál la peor opción. Los resultados obtenidos sugieren que algo más de tres cuartas partes $(77,8 \%)$ del alumnado participante es capaz de determinar cuál es el texto plagiado: texto "B" (tabla 1 ).

El $72,3 \%$ acertadamente responde que el mejor fragmento para presentar en un trabajo académico es el " $C$ ".

El $68 \%$ es capaz de determinar, con acierto, que el peor fragmento para entregar como trabajo académico es el " $B$ ".

La recodificación y posterior sumatorio de los datos obtenidos en esta pregunta -explicado en la metodología- posibilitan generar un IRP que arroja los siguientes resultados:

- casi la mitad de la muestra $(49,2 \%)$ acertó en las tres preguntas: IRP alto;

- un $27,1 \%$ respondió correctamente dos preguntas: IRP medio;

- un $14,5 \%$ respondió correctamente una de las tres preguntas: IRP bajo;

- un 8,6\% no contestó acertadamente ninguna de las preguntas: IRP Nulo.

Se ha realizado la prueba t-student para analizar la relación estadística entre el IRP y el género, y los resultados obtenidos demuestran la inexistencia de dicha asociación (significación bilateral $=0,487$ ).

\subsection{Conocimiento de las citas y referencias bibliográ- ficas}

El $64,9 \%$ del alumnado encuestado fue capaz de determinar que la definición aportada correspondía a una cita académica (tabla 2). El 91,5\% supo determinar que la información que se le presentaba, en otra pregunta, era una referencia

Tabla 1. Reconocimiento de plagio en textos académicos

\begin{tabular}{|l|c|l|l|l|}
\hline \multicolumn{2}{|c|}{$\begin{array}{c}\text { ¿Cuál de los tres fragmentos representa un } \\
\text { plagio del original? (\%) }\end{array}$} & \multicolumn{2}{c|}{$\begin{array}{c}\text { ¿Cuál es el mejor fragmento para entregar } \\
\text { como parte de un trabajo académico? (\%) }\end{array}$} & $\begin{array}{c}\text { ¿Cuál es el peor fragmento para entregar } \\
\text { como parte de un trabajo académico? (\%) }\end{array}$ \\
\hline Texto A (Parafraseado sin cita) & 7,7 & Texto A (Parafraseado sin cita) & 20,0 & Texto A (Parafraseado sin cita) \\
\hline Texto B (Plagio literal) & 77,8 & Texto B (Plagio literal) & 6,5 & Texto B (Plagio literal) \\
\hline Texto C (Texto correctamente citado) & 6,5 & Texto C (Texto correctamente citado) & 72,3 & Texto C (Texto correctamente citado) \\
\hline No lo sé & 8,0 & No lo sé & 1,2 & No lo sé \\
\hline
\end{tabular}


Tabla 2. Reconocimiento de citas y referencias bibliográficas en textos académicos

\begin{tabular}{|l|c|l|c|}
\hline $\begin{array}{c}\text { ¿A qué concepto corresponde esta definición: “incorporación dentro del texto de } \\
\text { conceptos, palabras o frases de un autor determinado identificando el linaje de } \\
\text { este y el año de publicación para que cualquier lector pueda localizar la fuente } \\
\text { utilizada"? (\%) }\end{array}$ & $\begin{array}{c}\text { Si en un trabajo académico encuentras: } \\
\begin{array}{c}\text { Novo, M. (1988). La educación ambiental: bases } \\
\text { éticas y metodológicas. Ginebra: Unesco. } \\
\text { ¿Qué es? (\%) }\end{array}\end{array}$ \\
\hline Referencia bibliográfica & 23,8 & Nota a pie de página & 2,0 \\
\hline Cita & 64,9 & Referencia bibliográfica & 91,5 \\
\hline Nota a pie de página & 8,6 & Paráfrasis bibliográfica & 3,3 \\
\hline Bibliografía & 2,0 & Cita & 0,0 \\
\hline No lo sé & 0,7 & No lo sé & 3,3 \\
\hline
\end{tabular}

bibliográfica redactada con arreglo a la normativa APA (tabla 2).

En cuanto al ICCRB:

- el 60,9\% de la muestra contestó acertadamente las dos preguntas: ICCRB alto;

- el 34,4\% acertó en una pregunta: ICCRB bajo;

- el 4,6\% erró en las dos preguntas: ICCRB nulo.

En cuanto a la relación entre el ICCRB y el género, la prueba t-student demuestra la inexistencia de una relación estadísticamente significativa entre ambas variables (sig. bilateral $=0,326$ ).

\subsection{Comisión de plagio académico}

Las prácticas más extendidas (tabla 3) son las calificadas en la bibliografía como "plagio collage" (Comas-Forgas, 2009), esto es, componer un trabajo académico a partir de la copia de diversos fragmentos de texto, bien sea de fuentes digitales bien sea de fuentes impresas, y su inserción en un documento en el que hay alguna parte de aportación propia, sin citar su origen. Entre las acciones menos repetidas, sobresale:

- descargar un trabajo completo de internet y entregarlo como propio;

- presentar un trabajo elaborado únicamente a partir de fragmentos copiados de internet;

- presentar un trabajo elaborado y previamente entregado en cursos o convocatorias anteriores.

Ahondando en la explotación de resultados, se ha efectuado el sumatorio de respuestas de las siete acciones analizadas con el fin de generar el ICPA. El resultado de la

Tabla 3. Frecuencia de respuesta para las 7 acciones constitutivas de plagio académico

\begin{tabular}{|l|c|c|c|c|c|}
\hline Acción & $\begin{array}{c}\text { Nunca } \\
\text { (\%) }\end{array}$ & $\begin{array}{c}\mathbf{1} \text { a } \mathbf{2} \\
\text { veces (\%) }\end{array}$ & $\begin{array}{c}\mathbf{3} \text { a 5 } \\
\text { veces (\%) }\end{array}$ & $\begin{array}{c}\mathbf{6} \text { a 10 } \\
\text { veces (\%) }\end{array}$ & $\begin{array}{c}\text { Más de 10 } \\
\text { veces (\%) }\end{array}$ \\
\hline 1 & 80,7 & 17,7 & 1,6 & & \\
\hline 2 & 76,9 & 21,2 & 1,2 & 0,3 & 0,3 \\
\hline 3 & 37,0 & 32,3 & 22,4 & 5,0 & 3,4 \\
\hline 4 & 86,8 & 12,3 & 0,3 & 0,3 & 0,3 \\
\hline 5 & 43,6 & 31,2 & 20,6 & 2,8 & 1,9 \\
\hline 6 & 86,3 & 7,8 & 3,4 & 1,9 & 0,6 \\
\hline 7 & 51,6 & 34,3 & 11,6 & 1,9 & 0,6 \\
\hline
\end{tabular}

Tabla 4. Valores obtenidos mediante la prueba $t$ para muestras independientes para el análisis de relaciones entre el ICPA y el género

\begin{tabular}{|l|l|c|c|c|c|c|}
\hline & Género & $\mathbf{N}$ & $\bar{x}$ & $\mathbf{t}$ & gl & $\begin{array}{c}\text { Significación } \\
\text { (bilateral) }\end{array}$ \\
\hline \multirow{2}{*}{ ICPA } & Mujer & 162 & 10,18 & & & \\
\cline { 2 - 7 } & Hombre & 151 & 10,72 & & & 0,1520 \\
\hline
\end{tabular}

$\mathrm{N}=\mathrm{n}$ ㅇ de alumnos de la muestra; $\overline{\mathrm{x}}=$ =media; $\mathrm{t}=$ valor obtenido; gl=grados de libertad.

agrupación genera una media de respuesta de 10,46 con una desviación típica de 3,38 (siendo el valor mínimo 7 y el máximo 26).

\subsection{Asociación entre el índice de comisión de plagio académico y el género}

Los hombres presentan medias de perpetración más altas que las mujeres, aunque no se constata una relación estadísticamente significativa (tabla 4).

\subsection{Asociación entre el índice de comisión de plagio académico y el índice de reconocimiento de plagio}

Los alumnos con un bajo IRP son los que tienen un ICPA más alto, lo cual podría resultar esperable. Ahora bien, el segundo grupo con un ICPA mayor es el que conforman aquellos que han obtenido un IRP más alto; es decir, aquellos alumnos que saben reconocer lo que supone un texto plagiado presentan tasas de plagio bastante elevadas, si lo comparamos con el resto (tabla 5). Sea como sea, no existe una relación significativamente estadística entre ambas variables ya que el análisis de la varianza realizado (anova de un factor) arroja una significación de 0,186.

Tabla 5. Medias de ICPA asociadas a los niveles de IRP

\begin{tabular}{|l|r|r|}
\hline \multicolumn{1}{|c|}{ IRP } & N & $\bar{X}$ ICPA \\
\hline Nulo & 26 & 11,53 \\
\hline Bajo & 43 & 9,95 \\
\hline Medio & 88 & 10,12 \\
\hline Alto & 156 & 10,62 \\
\hline
\end{tabular}

$\bar{X} \quad$ ICPA $=$ media del ICPA 
Tabla 6. Medias de ICPA asociadas a los niveles de ICCRB

\begin{tabular}{|l|c|c|}
\hline \multicolumn{1}{|c|}{ ICCRB } & N & $\overline{\mathbf{X}}$ ICPA \\
\hline Nulo & 17 & 11,85 \\
\hline Bajo & 104 & 10,20 \\
\hline Alto & 185 & 9,91 \\
\hline
\end{tabular}

X ICPA $=$ media del ICPA

\subsection{Asociación entre el índice de comisión de plagio académico y el índice de conocimiento de las citas y referencias bibliográficas}

El alumnado que tiene un ICCRB más alto presenta unos niveles de ICPA más bajos y a la inversa. Los datos parecen tener bastante lógica: a mejor conocimiento de las citas y referencias, menor propensión a plagiar. Aun así, los resultados obtenidos no son estadísticamente significativos dado que el análisis realizado de la varianza (anova de un factor) arroja una significación de 0,337.

Los alumnos que saben reconocer lo que es un plagio presentan tasas de plagio bastante elevadas, si lo comparamos con el resto

\section{Limitaciones, discusión y conclusiones}

El trabajo aquí presentado adolece de ciertas limitaciones que los autores consideran necesario publicitar: por un lado, la muestra está compuesta exclusivamente por alumnado de dos titulaciones de un área determinada en su último curso de carrera y este hecho invalida la posibilidad de generalizar los resultados obtenidos a un espectro mayor de la población universitaria española. Aun así, las características de la muestra -que sean alumnos a punto de graduarse y salir al mercado laboral y/o continuar con una formación de postgrado- aporta datos altamente significativos acerca de qué competencias adquiere el alumnado de administración y dirección de empresas (GADE) y del grado de economía (GECO) en la universidad -en lo tocante a la elaboración de trabajos académicos desde una perspectiva ética y con arreglo a las normas de comunicación científica-, y da pistas de cuán preparados están para su entrada en el mercado laboral -o su continuidad en el ámbito académico- y desarrollarse profesionalmente de manera íntegra y honesta.

En segundo lugar, el hecho de basar el estudio en métodos de encuesta puede provocar cierto sesgo en los datos obtenidos por factores de deseabilidad social (tendencia a atribuirse cualidades y comportamientos socialmente deseables y rechazar los socialmente no deseables). Los autores son conscientes de que el fenómeno del plagio puede abordarse con otras técnicas, una de las cuales, implica el uso de programas de detección para calibrar con mayor precisión la extensión del fenómeno. Se ha descartado dicha opción porqué los programas que presentan unos niveles de fiabilidad aceptables, aunque sin ser infalibles (WeberWulff et al., 2013), son de pago y el coste de la licencia para la muestra con que se trabajó, junto al hecho de ser ésta una aproximación incipiente que se pretende ampliar y mejorar en el futuro, hacían inviable esta posibilidad.

Los resultados alcanzados por este trabajo ponen de manifiesto que una parte importante del alumnado de último curso de las carreras del área de Economía tiene serias dificultades para reconocer lo que supone un plagio en la composición escrita. Es sorprendente que, después de haber cursado al menos 7 semestres en la universidad, casi la mitad de la muestra con la que se trabajó no fuese capaz de contestar acertadamente las tres preguntas referidas al reconocimiento del plagio académico. Algo se debe estar haciendo mal desde las instituciones académicas, sobre todo, cuando estos datos son altamente parejos con los resultados obtenidos por estudios internacionales de análogas características (Roig, 1997; Moon, 2002; Guedes; Gomes-Filho, 2015).

En la misma línea, aunque con unos resultados un tanto más positivos que los anteriores, van las apreciaciones dirigidas a comentar el resultado del reconocimiento de las citas y referencias. Que 4 de cada 10 alumnos de último curso encuestados haya errado en al menos una de las dos preguntas de reconocimiento de las citas y las referencias bibliográficas da bastante qué pensar y lleva a los autores a cuestionarse cómo habrán hecho los trabajos académicos durante su trayectoria en la universidad y en qué disposición están estos alumnos para afrontar la elaboración del trabajo fin de grado (TFG) y, en un futuro muy próximo, su labor profesional y/o continuidad en otros estudios superiores.

Una parte importante del alumnado de último curso de las carreras del área de Economía tiene serias dificultades para reconocer lo que supone un plagio en la composición escrita

Los datos relativos a la comisión de plagio académico ponen de manifiesto que esta práctica está ampliamente extendida en las aulas universitarias: sólo el $22,4 \%$ de la muestra afirmó no haber cometido plagio durante el curso académico anterior a la administración del cuestionario. Esta cifra es muy parecida a la obtenida por trabajos nacionales centrados en alumnado universitario (Comas-Forgas, 2009) y también entre alumnado de educación secundaria obligatoria y bachillerato (Sureda-Negre; Comas-Forgas; Oliver-Trobat, 2015). El fenómeno del plagio académico no nace en la universidad, pero en ella tampoco parece que se corrija. Es interesante, en este punto, destacar el hecho que existe un $6,4 \%$ de los participantes que podemos calificar como de "plagiadores accidentales", siguiendo la clasificación de Park (2003). Se trata de alumnos que son incapaces de reconocer el plagio y que a la vez cometen acciones plagiarias. Este dato, sumado al 22,4\% -porcentaje de "no plagiadores"supone que el resto $(71,2 \%)$ cometió al menos una acción constitutiva de plagio teniendo cierta o plena conciencia de lo que estaba haciendo. Son datos ciertamente alarmantes.

De manera general, a la luz del presente estudio, no se demuestra que exista una relación entre la capacidad para reconocer cuándo un texto está plagiado y la comisión de este tipo de falta. De la misma manera, no se puede concluir que 
el hecho de saber reconocer qué es una cita y una referencia bibliográfica represente que se plagie menos. Ello invita a pensar que el conocimiento de lo que es y no es plagio y de las normas de citación no es suficiente para atajar el problema aunque a buen seguro ayuda.

Es imprescindible que desde las instituciones educativas se luche contra todo tipo de conducta deshonesta que vaya contra los valores de la probidad y la honradez. Los centros educativos y universidades deben incluir entre sus objetivos luchar contra esta plaga (Hexham, 2013) y formar a ciudadanos íntegros y honestos. No se puede expresar de forma más clara de lo que lo hizo Moreno (1999):

La escuela es el primer 'campo de prácticas' del fraude y la corrupción; el primer escenario público en el que se adquiere y desarrolla una serie de conductas y 'habilidades' que más adelante se utilizarán en las relaciones con otras instituciones sociales, a saber, el mercado laboral, la Seguridad Social, la Hacienda Pública, etc. Y, además, la escuela también es el primer espacio en el que se aprende a disculpar, justificar y hasta a legitimar tales conductas y habilidades" (p. 72).

\section{Bibliografía}

Christakis, Erika; Christakis, Nicholas (2012). "Harvard cheating scandal: Is academic dishonesty on the rise". Time ideas, 4 Sept. http://ideas.time.com/2012/09/04/harvard-cheatingscandal-is-academic-dishonesty-on-the-rise

Cizek, Gregory (1999). Cheating on tests: How to do it, detect it, and prevent it. Routledge. ISBN: 9780805831443

Comas-Forgas, Rubén (2009). El ciberplagio y otras formas de deshonestidad académica entre el alumnado universitario. Tesis doctoral (no publicada). Palma Mallorca, España: Universidad de las Islas Baleares.

Comas-Forgas, Rubén; Sureda-Negre, Jaume; Salva-Mut, Francesca (2010). "Academic plagiarism prevalence among Spanish undergraduate students: an exploratory analysis". Biochemia medica, v. 20, n. 3, pp. 301-306.

http://dx.doi.org/10.11613/bm.2010.038

DeGeeter, Michelle; Harris, Kira; Kehr, Heather; Ford, CaroIyn; Lane, Daniel; Nuzum, Donald; Compton, Cinthya; Gibson, Withney (2014). "Pharmacy students' ability to identify plagiarism after an educational intervention". American journal of pharmaceutical education, v. 78, n. 2.

http://dx.doi.org/10.5688/ajpe78233

Domínguez-Aroca, María-Isabel (2012). "Lucha contra el plagio desde las bibliotecas universitarias". El profesional de la información, v. 21, n. 5, pp. 498-503.

http://dx.doi.org/10.3145/epi.2012.sep.08

Guedes, Diego-Oliveira; Gomes-Filho, Douglas-Leonardo (2015). "Percepção de plágio acadêmico entre estudantes do curso de odontologia". Revista bioética, v. 23, n. 1, pp. 139-148. http://dx.doi.org/10.1590/1983-80422015231054

Hallak, Jacques; Poisson, Muriel (2002). Ethics and corruption in education. Paris: Unesco.

http://www.unesco.org/iiep/PDF/Forum15.pdf
Hallak, Jacques; Poisson, Muriel (2007). "Fraude académico, acreditación y garantía de calidad: lecciones aprendidas del pasado y retos para el futuro". En: Sanyal, Bikas; Tres, Joaquín (ed.). La educación superior en el mundo - 2007. Acreditación para la garantía de la calidad: ¿Qué está en juego? España: Mundi-Prensa. ISBN: 848476303 X http://goo.gl/LMcpUJ

Hexham, Irving (2013). The plague of plagiarism: Academic plagiarism defined. Irving Hexham (ResearchGate profile). https://goo.gl/vXODSy

Humphreys, Louise (2013). "The internet as a challenge to HE: Implications for plagiarism \& information literacy" (poster). Innovative practice in higher education, v. 1, n. 3. http://journals.staffs.ac.uk/index.php/ipihe/article/viewFile/40/85

Hurlbert, Janet-McNeil; Savidge, Cathleen; Laudenslager, Georgia (2003). "Process-based assignments: How promoting information literacy prevents plagiarism". College \& undergraduate libraries, v. 10, n. 1, pp. 39-51.

Moon, Young-in (2002). "Korean university students' awareness of plagiarism in summary writings". Language research, v. 38, n. 4, pp. 1349-1365.

http://s-space.snu.ac.kr/bitstream/10371/90765/1/12.\%20 2225589.pdf

Moreno, Juan-Manuel (1999). “Con trampa y con cartón. El fraude en la educación, o cómo la corrupción también se aprende". Cuadernos de pedagogía, n. 283, pp. 71-77. http://cedoc.infd.edu.ar/upload/Con_trampa.....PDF

Morey, Mercè (2011). Anàlisi de l'alfabetització informacional entre l'alumnat de la Universitat de les Illes Balears. Tesis doctoral (no publicada). Palma Mallorca, España: Universidad de las Islas Baleares.

Park, Chris (2003). “In other (people's) words: Plagiarism by university students - literature and lessons". Assessment \& evaluation in higher education, v. 28, n. 5, pp. 471-488.

http://www.lancaster.ac.uk/staff/gyaccp/caeh_28_5_02lores. $p d f$

http://dx.doi.org/10.1080/02602930301677

Poisson, Muriel (2010). Corruption and education. Paris: International Institute for Educational Planning, Unesco. ISBN: 978928031342

Roig, Miguel (1997). "Can undergraduate students determine whether text has been plagiarised?". Psychological record, v. 47, n. 1, pp. 113-122.

http://goo.gl/vFMNvH

Sureda-Negre, Jaume; Comas-Forgas, Rubén; Oliver-Trobat, Miquel-Francesc (2015). "Plagio académico entre alumnado de secundaria y bachillerato: diferencias en cuanto al género y la procrastinación". Comunicar, v. 22, n. 44, pp. 103-111. http://dx.doi.org/10.3916/c44-2015-11

Weber-Wulff, Deborah; Möller, Christopher; Touras, Jannis; Zincke, Elin (2013). "Plagiarism detection software test (2013)". Abgerufen am, n. 12.

http://plagiat.htw-berlin.de/wp-content/uploads/Testbericht2013-color.pdf 